\title{
Fahr's Syndrome due to Hypoparathyroidism Following Thyroidectomy
}

\author{
Felipe Bozi Soares ${ }^{\mathrm{a}}$, Fabio Ferreira Amorim ${ }^{\mathrm{a}, \mathrm{b}, \mathrm{c}, \mathrm{e}}$, Adriell Ramalho Santana ${ }^{\mathrm{a}}$, \\ Edmilson Bastos de Moura ${ }^{c}$, Silviano Barretto Margalho ${ }^{\mathrm{b}}$, \\ Alethea Patricia Pontes Amorimc, Marcelo de Oliveira Maiac, \\ Flavio Palhano de Jesus Vasconcelos ${ }^{\mathrm{c}, \mathrm{d}}$
}

\begin{abstract}
Fahr's syndrome is a rare, degenerative, and neuropsychiatric disorder characterized by seizures, extrapyramidal, and neuropsychiatric symptoms as a result of symmetric and bilateral calcifications of the nucleus pallidus, the putamen, the dentate nucleus of the cerebellum, and the hemispheric white matter at the base of the skull. The female patients, who has 53-year-old, was admitted in the intensive care unit with status epilepticus for 24 hours associated to acute respiratory failure due to aspiration pneumonia. She had undergone a thyroidectomy 22 years ago, when she started involuntary lasting muscular contractions of the upper extremities, which progressed to convulsions throughout the years. There were Chvostek and Trousseau signs. Laboratory tests showed ionic calcium of $0.55 \mathrm{mmol} / \mathrm{L}$. Computed tomography scan of the brain showed intracranial symmetrical calcifications of the basal nuclei, semioval center, occipital, and cerebellar cortex. According to the clinical presentation, examination findings, and subsequent investigation exams, our case was diagnosed as Fahr's syndrome due to post-thyroidectomy hypoparathyroidism. Fahr's syndrome should be considered in patients with neuropsychiatric disturbances and seizure disorder, especially following thyroidectomy.
\end{abstract}

Keywords: Fahr's syndrome; Basal ganglia calcification; Bilateral intracranial calcification; Hypoparathyroidism; Parathyroid diseases; Hypocalcemia; Epilepsy; Thyroidectomy

\footnotetext{
Manuscript accepted for publication March 22, 2013

${ }^{a}$ Faculty of Medicine, Escola Superior de Ciencias da Saude, Brasilia, DF, Brazil

${ }^{\mathrm{b}}$ Adult Intensive Care Unit, Hospital Regional de Samambaia, Brasilia, DF, Brazil

${ }^{c}$ Adult Intensive Care Unit, Hospital Santa Luzia, Brasilia, DF, Brazil

${ }^{\mathrm{d} D i v i s i o n}$ of Endocrinology, Hospital Regional de Sobradinho, Brasilia, DF, Brazil

${ }^{\mathrm{e}}$ Corresponding author: Fabio Ferreira Amorim, Coordenacao de Pesquisa e Comunicacao Cientifica. SMHN Quadra 03, conjunto A, Bloco 1, Edifício FEPECS, Brasilia, DF, Brazil.

Email: ffamorim@gmail.com
}

doi: http://dx.doi.org/10.4021/jmc1252e

\section{Introduction}

Fahr's syndrome is a rare, degenerative, and neuropsychiatric disorder characterized by seizures, extrapyramidal and neuropsychiatric symptoms as a result of symmetric and bilateral calcifications of the nucleus pallidus, the putamen, the dentate nucleus of the cerebellum (striato-pallido-dentate calcinosis) and the hemispheric white matter at the base of the skull $[1,2]$. This syndrome is mostly associated with a disorder of calcium and phosphate metabolism, especially to hypoparathyroidism (HPT) [2-5], but can also be attributed to other different etiologies, including infectious, metabolic, and genetic diseases [1].

The clinical manifestations can be paresthesias, seizures, myoclonus, tetany, parkinsonism, and neuropsychiatric disorders (such as cognitive and psychotic disorders) $[1,5-7]$. Early treatment, if instituted correctly, can reverse neurological symptoms and prevent irreversible alterations thus emphasizing the need to recognize early clinical manifestations $[1,2,5,6]$.

The current article reports a case of Fahr's syndrome due to hypoparathyroidism following thyroidectomy.

\section{Case Report}

The female patient, who was 53-years-old and brown skinned, was admitted to the intensive care unit (ICU) with a condition of status epilepticus for 24 hours associated to acute respiratory failure due to aspiration pneumonia. Regarding her past medical history, the patient had undergone a thyroidectomy 22 years ago, when she started involuntary lasting muscular contractions of the upper extremities, which progressed to convulsions throughout the years. She also had episodes of paresthesia of the lower extremities and was a long-term user of Phenytoin. Her physical examination revealed poor general condition, pallor, hydrated, afebrile, anicteric and acyanotic. Her blood pressure was 125 $\times 79 \mathrm{mmHg}$ and her heart rate was 78 beats per min. Pulmonary auscultation revealed crackles in right hemithorax. There were spastic tetraparesis with muscle shortening and 


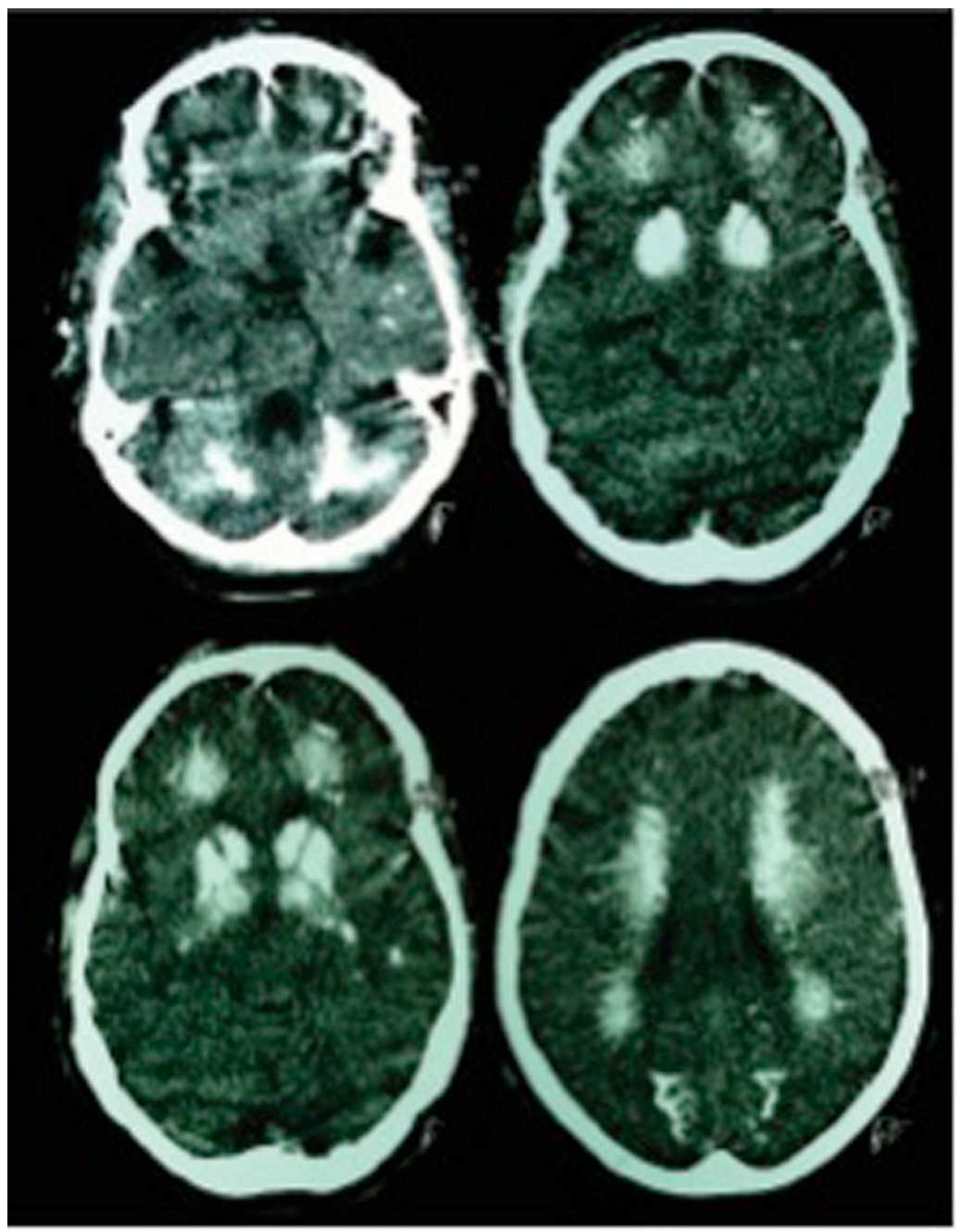

Figure 1. Non-contrast computed tomography scan of the brain showing diffuse calcifications in cerebellum, basal ganglia, semioval centers, thalamus, frontal, temporal, and occipital white matter.

stiffness of respiratory and shoulder girdle muscles, also Chvostek and Trousseau signs.

On admission, the laboratory tests showed hypocalcemia (total calcium of $3.2 \mathrm{mg} / \mathrm{dL}$ (normal level $8.4-10.5$ ), and ionic calcium $0.55 \mathrm{mmol} / \mathrm{L}$ (normal level $1.12-1.23$ $\mathrm{mmol} / \mathrm{L})$ ). White blood cell count, and hemoglobin level were $15,500 / \mathrm{mm}^{3}$, and $9.5 \mathrm{~g} / \mathrm{dL}$, respectively. The levels of urea nitrogen and creatinine were normal $(22 \mathrm{mg} / \mathrm{dL}$ and 0.9 $\mathrm{mg} / \mathrm{dL}$, respectively). The parathormone (PTH) level was $1.3 \mathrm{pg} / \mathrm{mL}$ (normal level $15-65 \mathrm{pg} / \mathrm{mL}$ ). Chest radiograph showed alveolar infiltrate in the right lower lobe. Computed tomography scan of the brain showed intracranial symmetrical calcifications of the basal nuclei, semioval center, occipital, and cerebellar cortex, and no compressive effect (Fig. 1). According to the clinical presentation, examination findings, and subsequent investigation exams, our case was diagnosed as Fahr's syndrome due to post-thyroidectomy HPT.

Upon admission to the ICU, she was placed on mechani- cal ventilation. She was kept sedated and initiated treatment with intravenous calcium gluconate, and ampicilin/sulbactam. Attempts were made to suspend the sedation, but the patient kept presenting global muscle stiffness. On the third day, we initiated oral calcium and calcitriol. On the sixth day, we took out the sedation and the patient showed active movement but with loss of muscle strength and muscle shortening, with total calcium level of $9.3 \mathrm{mg} / \mathrm{dL}$. The mechanical ventilation was withdrawn on the ninth day, after reduction of the spastic hypertonia of respiratory muscles and presented active movement of the extremities with discrete muscular hypertonia. On the thirteenth day, she was discharged stable but with mild disorientation.

\section{Discussion}

HPT may be associated with a broad spectrum of clinical 
Table 1. Conditions Associated With Basal Ganglia Calcification on Neuroimaging

\section{Classification}

Endocrine

Tumoral

Hypoxic and vascular

Inflammatory/Infectious

Toxic

Genetic

Other

\section{Cause}

Hypoparathyroidsm, pseudohypoparathyroidism pseudopseudohypoparathyroidism, hypothyroidism and hyperparathyroidism.

Astrocytoma.

Ateriovenous malformation, calcified infarct, and ischemic encephalopathy.

Cytomegalovirus infection, tuberculosis, measles, toxoplasmosis neurobrucellosis, mumps, congenital rubella, neurocysticercosis, varicella, acquired immunodeficiency syndrome, coxsackie B infection, and systemic lupus erythematous.

Carbon monoxide intoxication, lead poisoning, hypervitaminosis D, methotrexate therapy, and radiotherapy.

Familial idiopathic basal ganglia calcification (Fahr's disease), idiopathic lenticulodentate calcification (Hastings-James syndrome), Cockayne's syndrome, Griscelli disease, MELAS (mitochondrial, myopathy, encephalopathy, lactic acidosis, and stroke) syndrome, MERRF (myoclonic epilepsy with ragged red fibers) syndrome, Kearns-Sayre syndrome, Leigh's disease, Sturge-Weber-Dimitri syndrome, Down's syndrome, lipoid proteinosis (Urbach-Wieth disease), carbonic anhydrase II deficiency syndrome, biopterin deficit, leukodistrophic diseases, arthrogryposis, and tuberous sclerosis.

Senility, malabsorption, motor neuron disease. manifestations [8]. One of these is Fahr's syndrome, that is characterized by seizures, extrapyramidal, and neuropsychiatric signs as a result of symmetric and bilateral calcifications of the nucleus pallidus, the putamen, the dentate nucleus of the cerebellum (striato-pallido-dentate calcinosis), and hemispheric white matter [1, 2]. In 1930, Karl Theodor Fahr, a German pathologist, first described this syndrome in a case of a man with seizures, and diffuse calcifications of brain vessels, and basal ganglia [9].

The prevalence of Fahr's syndrome is unknown, but an incidence of basal ganglia calcifications (BGC) ranging from $0.3 \%$ to $1.2 \%$ has been reported in routine radiological examinations [10, 11]. Kazis [10] analyzing 7040 computed tomography scan of the brain reported an incidence of $1.02 \%$ of symmetrical intracranial calcifications. In this study, all patients who had more extensive calcifications showed clear findings of HPT. Goswani et al [3] reported the occurrence of BCG in $73.8 \%$ in idiopathic HPT.

The pathogenic mechanisms of BGC are unknown, although it is believed to be due to poor calcium and phosphorus control in the HPT, associated to hypokalemia, and low calcium/phosphorus ratio [3]. Active role of PTH in basal ganglia physiology may also be involved [3]. On histopatho- logical study, Fahr's syndrome can be labeled as intracerebral small vessel and perivascular space mineralization primarily of basal ganglia, cerebellar structures and white brain matter [12], that consists mainly of hydroxyapatite of a nature similar to that found in bones, and other elements (such as, zinc, iron, and magnesium) enmeshed in a protein rich stroma without collagen or mucopolysaccharides [13, 14].

Intracranial calcifications are a common finding in neuroimaging. Although the most common cause of BGC are disorders of calcium and phosphate metabolism, more than fifty clinical conditions have been associated with BGC, including inflammatory, infectious, tumoral, endocrine, hypoxic, vascular, toxic, metabolic, and genetic disorders (Table 1) [1, 7]. Among these conditions, the endocrine diseases, especially HPT, are the most common causes of Fahr's syndrome [2-5].

About half of the patients with BGC exhibit neuropsychiatric manifestations and deficits. The most common neurological symptoms are headache, vertigo, movement disorders (such as Parkinson's tremor, dystonia, and cerebellar ataxia), myoclonus and seizures [1]. Other neurological symptoms consist of stroke like events, speech disorders, coma, cognitive impairment, and syncope $[1,6,7,15,16]$. 
The psychiatric symptoms are usually more prominent than the neurologic symptoms in the onset of the disease $[4,5]$. Forty percent of patients with BGC themselves present with psychiatric symptoms at the beginning of the disease, and usually precede neurological manifestations [4, 5]. The psychiatric symptoms may be composed of a wide variety of symptoms ranging from depression to psychosis $[2,4,5$, 16, 17]. Among these, cognitive and psychotic disorders are most prominent $[4,5]$.

In the presence of BGC, especially with symptoms, laboratory investigation should be performed to assess the occurrence of HPT. The typical findings in untreated HPT are low circulation PTH levels, hypocalcemia, hyperphosphatemia, low levels of 1,25-dihydroxyvitamin D, and relatively high urinary calcium excretion. Laboratory evaluation also should include serum creatinine, and magnesium levels. The finding of hypocalcemia associated to undetectable PTH levels is sufficient to confirm diagnosis, since it rules out all other causes of hypocalcemia, which would lead to an increase activity of the parathyroid gland [9, 16, 18].

As regards the present case, the patient had undergone a previous thyroidectomy. The most common cause of HPT is inadvertent damage or removal of the parathyroid glands during thyroidectomy $[6,8,19,20]$. After total thyroidectomy, transient HPT can be observed in as high as $10 \%$ of patients. Most of these patients recover parathyroid function within several months, however about $4.4 \%$ develop permanent HPT [8]. In postoperative HPT, BGC will develop in untreated patients after a median of 17 years [6].

The treatment of Fahr's syndrome is directed to the identifiable cause [19]. Especially in HPT, the early treatment can prevent calcifications and neurophysiological disorders [2, 3, 16]. Goswami [3] reported a reduced risk of BCG progression with treatment to restore calcium/phosphorus ratio in patients with idiopathic HPT. In symptomatic patients, seizures show poor response to anticonvulsants, and resolution when normal plasma calcium level is reached [2]. Parkinsonism is also characterized by resistance to standard treatment, and in early cases may respond to calcium therapy [2]. The psychiatric symptoms usually improve with normalization of calcemia, although the dementia has the worst response [2]. It is noteworthy that the prognosis is variable, cannot be predicted and is unrelated to the extent of calcifications [16]. Death is usually due to neurological deterioration [16].

The standard treatment of HPT involves calcium supplementation and vitamin D or 1, 25-dihydroxyvitamin D (calcitriol) $[9,18,19]$. The treatment goals include symptoms control, and maintaining serum calcium in the low to normal range, serum phosphorus within normal limits, 24-hour urine calcium under $7.5 \mathrm{mmol} /$ day (300 mg/day) and the calcium-phosphate product under $55 \mathrm{mg} / \mathrm{dL}$ (4.4 mmol/L) [8]. It is notable that HPT is one of the few endocrine diseases that are not routinely treated by the replacement of the miss- ing hormone, although subcutaneous synthetic PTH could be used in refractory HPT [9, 18, 19].

Despite its rarety, the possibility of Fahr's syndrome should be considered in patients with neuropsychiatric disturbances and seizure disorder. In presence of BGC, HPT should be investigated, especially in patients who had undergone thyroidectomy. This case report stresses the major benefits of calcium supplementation and calcitriol, even with the patient being in advanced stage of disease.

\section{Acknowledgement}

FBS is a research scholar from FEPECS (Fundacao de Ensino e Pesquisa da Secretaria de Saude do Distrito Federal), Brasilia, DF, Brazil. ARS is a research scholar from $\mathrm{CNPq}$ (Conselho Nacional de Desenvolvimento Cientifico e Tecnologico), Brasilia, DF, Brazil.

\section{Declaration}

Study conducted at the Adult Intensive Care Unit, Hospital Regional de Samambaia, Brasilia, DF, Brazil.

\section{References}

1. Swami A, Kar G. Intracranial hemorrhage revealing pseudohypoparathyroidism as a cause of fahr syndrome. Case Rep Neurol Med. 2011;2011:407567.

2. Ramisa J, Ibanez AE, Irigoína RR, Artigasa CF, Comasa LM. Extrapyramidal symptoms due to calcinosiscerebri in a patient with unknown primary hypoparathyroidism. Endocrinol Nutr. 2012;59(1):69-71.

3. Goswami R, Sharma R, Sreenivas V, Gupta N, Ganapathy A, Das S. Prevalence and progression of basal ganglia calcification and its pathogenic mechanism in patients with idiopathic hypoparathyroidism. Clin Endocrinol (Oxf). 2012;77(2):200-206.

4. Srivastava S, Bhatia MS, Sharma V, Mahajan S, Rajender G. Fahr's Disease: An Incidental Finding in a Case Presenting with Psychosis. Ger J Psychiatry. 2010;13(2):86-90.

5. Senoglu M, Tuncel D, Orhan FO, Yuksel Z, Gokçe M. Fahr's Syndrome: A Report of Two Cases. Firat Tip Dergisi. 2007;12(1):70-72.

6. Castro MC, Singer BH. Agricultural settlement and soil quality in the Brazilian Amazon. Popul Environ. 2012;34(1):22-43.

7. Baba Y, Broderick DF, Uitti RJ, Hutton ML, Wszolek ZK. Heredofamilial brain calcinosis syndrome. Mayo Clin Proc. 2005;80(5):641-651.

8. De Sanctis V, Soliman A, Fiscina B. Hypoparathyroid- 
ism: from diagnosis to treatment. Curr Opin Endocrinol Diabetes Obes. 2012;19(6):435-442.

9. Fahr TK. Idiopatische Verkalkung der Hirngefasse. Zentralblallg Path u path anat 1930;50:129-133.

10. Kazis AD. Contribution of CT scan to the diagnosis of Fahr's syndrome. Acta Neurol Scand. 1985;71(3):206211.

11. Fenelon G, Gray F, Paillard F, Thibierge M, Mahieux F, Guillani A. A prospective study of patients with CT detected pallidal calcifications. J Neurol Neurosurg Psychiatry. 1993;56(6):622-625.

12. Unkrig S, Gullotta F, Madea B. Morbus Fahr--considerations on a case of sudden death. Forensic Sci Int. 2011;204(1-3):e12-15.

13. Konig P. Psychopathological alterations in cases of symmetrical basal ganglia sclerosis. Biol Psychiatry. 1989;25(4):459-468.

14. Smeyers-Verbeke J, Michotte Y, Pelsmaeckers J, Lowenthal A, Massart DL, Dekegel D, Karcher D. The chemical composition of idiopathic nonarteriosclerotic cerebral calcifications. Neurology. 1975;25(1):48-57.
15. Al-Jehani H, Ajlan A, Sinclair D. Fahr's Disease Presenting with Aneurysmal Subarachnoid Hemorrhage. J Clin Imaging Sci. 2012;2:27.

16. Rastogi R, Singh AK, Rastogi UC, Mohan C, Rastogi V. Fahr'ssyndrome: a rare clinico-radiologicentity. MJAFI 2011;67(2):159-161.

17. Gulsun M, Baykiz AF, Kabatas S, Belli H. Fahr's Syndrome - three cases presenting with psychiatric signs. Eur J Gen Med 2006;3(1):35-40.

18. Rizvi I, Ansari NA, Beg M, Shamim MD. Widespread intracranial calcification, seizures and extrapyramidal manifestations in a case of hypoparathyroidism. N Am J Med Sci. 2012;4(8):369-372.

19. Mitchell DM, Regan S, Cooley MR, Lauter KB, Vrla $\mathrm{MC}$, Becker CB, Burnett-Bowie SA, et al. Long-term follow-up of patients with hypoparathyroidism. J Clin Endocrinol Metab. 2012;97(12):4507-4514.

20. Paek SH, Lee YM, Min SY, Kim SW, Chung KW, Youn YK. Risk factors of hypoparathyroidism following total thyroidectomy for thyroid cancer. World J Surg. 2013;37(1):94-101. 